\title{
Are Newborn Outcomes Different for Term Babies Who Were Exposed to Antenatal Corticosteroids?
}

Alexandra McKinzie ${ }^{1}$, Ziyi Yang ${ }^{2}$, Evgenia Teal ${ }^{3}$, Joanne K. Daggy ${ }^{2}$, Robert S. Tepper ${ }^{4}$, Sarah K. Quinney ${ }^{1}$, Eli Rhoads ${ }^{4}$, Laura S. Haneline ${ }^{5}$, David M. Haas ${ }^{1}$

${ }^{1}$ Indiana University School of Medicine Department of Obstetrics and Gynecology, ${ }^{2}$ Indiana University School of Medicine Department of Biostatistics, ${ }^{3}$ Regenstrief Institute, Indianapolis, IN, ${ }^{4}$ Indiana University School of Medicine Division of Pediatric Pulmonology, ${ }^{5}$ Indiana University School of Medicine Division of Neonatology

Corresponding author: David Haas, MD, Department of OB/GYN, 550 N. University Blvd, UH2440, Indianapolis, IN 46202, (317)-880-3960, dahaas@iupui.edu

This study was funded, in part, with support from the Indiana Clinical and Translational Sciences Institute, funded in part by UL1TR002529 from the National Institutes of Health and by the Eunice Kennedy Shriver National Institute of Child Health and Human Development R01HD088014 (Haas). The content is solely the responsibility of the authors and does not necessarily represent the official views of the National Institutes of Health. Support also

This is the author's manuscript of the article published in final edited form as:

McKinzie, A., Yang, Z., Teal, E., Daggy, J. K., Tepper, R. S., Quinney, S. K., Rhoads, E., Haneline, L. S., \& Haas, D. M. (2021). Are Newborn Outcomes Different for Term Babies Who Were Exposed to Antenatal Corticosteroids? American Journal of Obstetrics and Gynecology. https://doi.org/10.1016/j.ajog.2021.04.251 
provided by the IU Dept. of OB/GYN to PREGMED: The Indiana University Center for Pharmacogenetics and Therapeutics Research in Maternal and Child Health. The authors have no conflicts of interest to disclose. 


\section{Condensation}

Term newborns who were exposed to antenatal corticosteroids for threatened preterm labor had higher rates of intensive care unit admissions and lower birthweights than babies not exposed to antenatal corticosteroids.

\section{Short title}

Outcomes for term newborns exposed to corticosteroids

\section{AJOG at a Glance}

A. Why was the study conducted?

- Women evaluated for threatened preterm labor are frequently given antenatal corticosteroids but then go on to deliver at term.

- It is unclear if this exposure to a single course of steroids has beneficial or harmful impacts on the term newborn.

B. What are the key findings?

- Compared to term newborns from women evaluated for threatened preterm labor but not given steroids, those newborns whose mothers were given steroids had higher rates of newborn intensive care unit admission and being small for gestational age, without any difference in rates of transient tachypnea of the newborn.

C. What does this study add to what is already known?

- The use of antenatal corticosteroids for women being evaluated for threatened preterm labor should be judicious, as this study demonstrates in a large, diverse population, that there may be associated adverse outcomes for exposed newborns who go on to deliver at term.

Keywords: antenatal corticosteroids, newborn outcomes, NICU admission, growth 


\begin{abstract}
Background: Antenatal corticosteroids improve newborn outcomes for preterm infants. However, predicting which women presenting for threatened preterm labor will have preterm infants is inaccurate and many women receive antenatal corticosteroids but then go on to deliver at term.
\end{abstract}

Objective: The purpose of this study was to compare the short-term outcomes of infants born at term to women who received betamethasone (BMZ) for threatened preterm labor to infants who were not exposed to BMZ in utero.

Study Design: We performed a retrospective cohort study of infants born at or after 37 weeks' gestational age (GA) to mothers diagnosed with threatened preterm labor during pregnancy. The primary neonatal outcomes of interest included transient tachypnea of the newborn (TTN), neonatal intensive care unit (NICU) admission, and small for gestational age (SGA), and were evaluated for their association with BMZ exposure while adjusting for covariates using multiple logistic regression.

Results: Of 5330 women, 1459 (27.5\%) women received $\mathrm{BMZ}$ at a mean GA of $32.2 \pm 3.3$ weeks. The mean age of women was $27 \pm 5.9$ years-old and the mean GA at delivery was $38.9 \pm 1.1$ weeks. Women receiving $\mathrm{BMZ}$ had higher rates of maternal comorbidities $(\mathrm{P}<0.001$ for diabetes, asthma, and hypertensive disorder) and were more likely to self-identify as white $(\mathrm{P}=0.022)$. BMZ-exposed neonates had increased rates of TTN, NICU admission, SGA, hyperbilirubinemia, and hypoglycemia (all P-values <0.05). Controlling for maternal characteristics and GA at delivery, BMZ exposure was not significantly associated with diagnosis of TTN (aOR 1.10, 95\% CI 0.80-1.51), though it was associated with more NICU 
admissions (aOR 1.49, 95\% CI 1.19-1.86) and higher odds of the baby being small for gestational age (SGA, aOR 1.78, 95\%CI 1.48 to 2.14 ).

Conclusions: Compared to women evaluated for preterm labor that did not receive BMZ, women receiving BMZ had infants with higher rates of NICU admission and SGA. While the benefits of BMZ to infants born preterm are clear, there may be negative impacts for infants delivered at term. 


\section{Background:}

A course of antenatal corticosteroids, such as betamethasone (BMZ), is typically administered to pregnant women between 24 - and 36+6/7-weeks' gestation at risk of delivery within 7 days ${ }^{1}$. Antenatal corticosteroids accelerate fetal lung development, subsequently leading to decreased rates of respiratory distress syndrome, respiratory support needed, and intensive care admission, as well as decreased incidence of complications common to preterm neonates, such as intraventricular hemorrhage, necrotizing enterocolitis, and neonatal death ${ }^{2}$.

Predicting which women presenting with threatened preterm labor will actually deliver before term, however, is still inexact due to inaccuracy of existing markers and the lack of understanding of underlying mechanisms ${ }^{3}$. Many women diagnosed with threatened preterm labor are given antenatal corticosteroids but do not deliver until they reach term ( $\geq 37$ weeks $)^{4}$.

It is unclear if exposure to antenatal corticosteroids for these term infants is beneficial or may lead to some harm. There are data demonstrating potential worsening lung function in infants exposed to antenatal corticosteroids. ${ }^{5,6}$ Thus, the imperative to give antenatal corticosteroids to women in threatened preterm labor quickly may not be prudent for those who go on to deliver at term.

The objective of this study was to compare the short-term outcomes of infants born at term (at least 37 weeks' gestation) to women who received BMZ injections for threatened preterm labor to infants who were not exposed to betamethasone in utero but whose mothers were also evaluated for threatened preterm labor.

\section{Materials and Methods:}


This study was a retrospective cohort study. The cohort consisted of babies born in Indianapolis, IN in Indiana University Health hospitals and Eskenazi Hospital from 2012-2019 at or after 37 weeks' gestation to mothers who had been evaluated for and diagnosed with threatened preterm labor at some point during pregnancy. These dates were chosen based on availability of electronic medical record data. The cohort was restricted to singleton infants born at term. Of primary interest was the comparison of infants born at term that were exposed to maternal BMZ therapy for threatened preterm labor and infants born at term whose mothers had been seen in an acute setting for threatened preterm labor but did not receive BMZ therapy.

Data for this study were collected from the electronic medical record (EMR) system data warehouse (Epic and Cerner) in de-identified fashion through the EMR data brokers at the Regenstrief Institute (Indianapolis, IN). Regenstrief then extracted the variables of interest from pharmacy records, discreet fields within the EMR, and diagnostic codes (ICD9/10). Maternal variables included age at time of infant delivery, race/ ethnicity, smoking status during pregnancy, date of BMZ injection, due date, maternal history of asthma, pre-existing or gestational diabetes, chronic hypertension, and hypertensive disorder of pregnancy. Short-term neonatal characteristics included gestational age at birth, date of birth, birth weight, birth length, birth head circumference, neonatal intensive care unit (NICU) admission, intubation, jaundice needing treatment, transient tachypnea of the newborn (TTN) diagnosis, oxygen support, treated hypoglycemia, and meconium aspiration syndrome diagnosis.

The primary neonatal outcomes of interest were TTN diagnosis, NICU admission, and SGA. The original analysis plan created prior to data extraction included TTN diagnosis, oxygen use, and birth length. However, after manual chart review, the hospital coding for oxygen use was not found to be accurate, thus NICU admission was included as a primary outcome instead. 
Also, SGA was included as the primary outcome instead of birth length as SGA is known to be more clinically meaningful. SGA was categorized by the published revised US birth weight reference published by Duryea et al. ${ }^{7}$ After data were extracted, a selection of random infants with exposure to $\mathrm{BMZ}$ and infants without exposure to $\mathrm{BMZ}(\sim 1 \%)$ had manual data abstraction for data validation/ verification to ensure that the diagnoses and codes were correct in the medical record. The study was approved by the local governing IRB and the scientific review group at the Regenstrief Institute Data Core.

Baseline characteristics were summarized for the entire cohort and by BMZ exposure status . Characteristics were compared between exposure groups using appropriate statistical tests (i.e. chi-square and t-tests). Multivariable logistic regression was employed to determine if BMZ exposure was independently associated with each neonatal outcome after adjusting for baseline characteristics. Regression models included factors known to contribute to newborn and long-term health outcomes (maternal age, socioeconomic status (insurance), race, and estimated gestational age (EGA) at delivery), and also factors that were found to significantly differ between exposure groups. To assess for the presence of collider bias with EGA at delivery, we repeated the regression excluding EGA at delivery as a variable. All data were incorporated into RedCap and analyzed using SAS Version 9.4 (Cary, NC).

Although an a priori sample size was not specifically calculated, based on initial database queries prior to data extraction, we anticipated data on at least 1500 women who received steroids and delivered past 37 weeks was estimated. Assuming that approximately $10 \%$ of babies were admitted to the NICU, a sample of 3000 women (half of whom received BMZ) would have $80 \%$ power to detect a change in the proportion with NICU admission from $10 \%$ to $13.7 \%$, corresponding to an adjusted Odds Ratio $(\mathrm{aOR})=1.43$, based on a logistic regression 
model adjusting for covariates. Even smaller odds ratios would be detectable if the proportion of non-exposed women was actually larger.

Human subjects' protection and data management: All investigators associated with this study passed the CITI human subjects certification. All data were stored in a secured RedCap database, analytic files were de-identified, and all computers used were password protected. Regenstrief data security policies were employed during data extraction. As a retrospective cohort study, a data safety monitoring board was not required.

Results:

Of 5330 women identified in the cohort, 1459 (27.4\%) received BMZ (Table 1). The majority of the population were not Hispanic or Latino (92.0\%) and were white $(71.4 \%)$. Approximately 55\% of women were insured by Medicaid or Government Insurance, while $43.4 \%$ had private insurance. The mean age of the women was $27 \pm 5.9$ years old. Women that did not receive $\mathrm{BMZ}$ were evaluated for threatened preterm labor at a mean gestational age of 33.0 \pm 5.0 weeks, while women in the BMZ group received the medication at a lower gestation age of 32.2 \pm 3.3 weeks. Women receiving BMZ had higher rates of maternal comorbidities ( $\mathrm{P}<.001$ for diabetes, asthma, and hypertensive disorder) and were more likely to self-identify as white $(\mathrm{P}=0.022)$ (Table 1$)$. Ethnicity did not differ between exposure groups and was not included in regression models. Maternal smoking status was extracted to use as a covariate but was missing for $49.5 \%$ of women thus was not included in any analyses. Neonatal characteristics considered as potential covariates included EGA at birth. Birth length, head circumference, and birth weight are provided as descriptive only as they are highly related to SGA (Table 2). Infants were delivered at a mean EGA of $38.9 \pm 1.1$ weeks with a mean birth weight of $3289.9 \pm 497.4$ grams, birth length of $52.0 \pm 77.6 \mathrm{~cm}$, and head circumference of 
$34.3 \pm 9.5 \mathrm{~cm}$. Women who received BMZ delivered babies at a slightly earlier EGA $(38.4 \pm 1.1$

vs. 39.1 \pm 1.1$)(\mathrm{P}<.001)$. Birth length and head circumference at birth did not significantly differ between exposure groups.

From the unadjusted analysis of outcomes, BMZ-exposed infants had higher rates of TTN diagnosis and NICU admission $(\mathrm{P}<0.05$, Table 2$)$. Based on the $10^{\text {th }}$ percentile birth weight threshold, $19.6 \%$ of infants in the BMZ exposure group were considered SGA, while $12.2 \%$ of infants in the non-exposed group were considered SGA $(\mathrm{P}<.001)$ (Table 2). BMZexposed infants also had higher rates of hyperbilirubinemia and hypoglycemia (all P-values $<0.05)$. Exposure groups did not significantly differ on intubation or meconium aspiration syndrome. After adjusting for multiple comparisons for the 4 secondary outcomes, BMZexposed infants were still more likely to be treated for hypoglycemia than non-exposed infants (Table 2).

After adjusting for maternal age, race, insurance, maternal conditions (diabetes, asthma, and hypertensive disorder) and EGA at delivery there was no significant difference in TTN diagnosis between BMZ exposure and BMZ non-exposure groups [Table 3, aOR 1.10 (95\% CI 0.80-1.51)]. Infants from women who self-identified their race as other/unknown were more likely to be diagnosed with TTN than those born to white women [aOR 1.70 (95\% CI 1.04-2.77)] and infants delivered at later gestational ages were less likely to have TTN diagnosis [aOR 0.74 (95\% CI 0.65-0.84)].

Infants from the BMZ exposure group were more likely to be admitted to the NICU [aOR 1.49 (95\% CI 1.19-1.86)], as were those born to mothers with maternal diabetes [aOR 1.50 (95\% CI 1.06-2.12)] or with Medicaid/ Government Insurance [aOR 1.42 (95\% CI 1.14-1.76)]. Infants whose mothers were black were less likely to be admitted to the NICU than those whose mothers 
were white [aOR $0.76(95 \%$ CI 0.58-0.99)]. Infants born at later gestational ages [aOR 0.63 (95\% CI 0.57-0.70)] were also less likely to be admitted to the NICU. (Table 3)

The adjusted analysis (Table 3) also demonstrated that infants in the BMZ exposure group were more likely to be classified as SGA than infants in the non-exposure group [aOR $1.78(95 \%$ CI 1.48, 2.14)]. Infants whose mothers self-identified their race as black [aOR 1.80 (95\% CI 1.50, 2.16)] or other/unknown [aOR 1.69 (95\% CI 1.24, 2.32)] were more likely to be SGA than those born to white women. Additionally, mothers covered by Medicaid/Government Insurance were more likely to have infants classified as SGA [aOR 1.29 (95\% CI 1.07, 1.53)].

Supplemental Table 1 displays the logistic regression for all three outcomes excluding EGA at delivery from the covariates. The associations with the three outcomes were not appreciably different.

\section{Structured Discussion/Comment:}

\section{Principal Findings}

Our results demonstrate that preterm antenatal exposure to BMZ for term-born neonates was associated with increased odds of NICU admission and SGA birthweights, but was not associated with increased odds of TTN after adjusting for clinical covariates. This points to a potential adverse impact of the current practice trend of administering antenatal corticosteroids with a low threshold to women being evaluated for threatened preterm birth.

2. Results in context

As so many women evaluated for threatened preterm birth do not deliver until term, the ability to prudently initiate interventions is important, given these results. In one study of 763 women who had unscheduled triage visits for symptoms of preterm labor, only $18 \%$ gave birth before 37 weeks of gestation and only $3 \%$ gave birth within 2 weeks of presenting with 
symptoms ${ }^{8}$. Better tools are needed for women presenting with threatened preterm labor to determine who will be at highest risk to deliver within 7 days and thus should receive antenatal corticosteroids ${ }^{1}$.

Our results, showing increased rates of SGA infants, are consistent with other literature showing the same adverse impact of multiple courses of antenatal steroids. ${ }^{9,10}$ These impacts have also been demonstrated in animal models ${ }^{4}$. Additionally, a similar study also found an increased rate of SGA infants exposed to antenatal corticosteroids who delivered at term (11\% vs $6 \%$ ), although that study's control group included all women delivering at term ${ }^{11}$. By limiting our control group to women evaluated for threatened preterm labor, we attempted to have a control group with similar pregnancy experiences to the women who received BMZ but delivered at term. While threatened preterm birth has been associated with fetal growth abnormalities $^{12}$, we have demonstrated that exposure to BMZ may increase rates of SGA beyond that for control women evaluated for threatened preterm birth. As it is known that SGA infants have higher rates of adverse health conditions later in life such as hypertension and metabolic issues, this antenatal exposure could be contributing to adverse health consequences.

Our results showing that black babies had lower rates of NICU admission, but higher rates of SGA were unexpected. It is known that there are disparities in newborn care and outcomes in both preterm and term infants by race. ${ }^{13,14}$ Given disparities in infant mortality in the U.S., it is important to further explore our results and the implications for care of black women with threatened preterm birth.

\section{Clinical Implications}

Given the known benefits to BMZ for preterm infants and the difficulty in predicting which women will actually deliver preterm, it is common for providers to administer antenatal 
corticosteroids in an effort not to miss the opportunity to give them before delivery and to try and get the maximal time benefit. However, as many of these babies will be born at term and may have adverse outcomes as we have demonstrated, it may be wise to use BMZ more judiciously for women who are evaluated for threatened preterm birth. This is particularly true as they are recommended for threatened late preterm birth as well.

\section{Research Implications}

Developing a more robust method to determine which women who present in threatened preterm labor will actually deliver within 1-2 weeks should continue to be a research priority. Additionally, a tool which would trigger a woman to be in a "high risk to deliver" category that would then be accompanied by BMZ administration could aid clinicians with this decision. Furthermore, better understanding the disparities in outcomes from these newborns and any differential impact that antenatal corticosteroids may have, accounting for social determinants of health, will be important. Replicating these findings in other populations will be important.

\section{Strengths and Limitation}

Given the large number of women in both groups and the diversity of our population, we believe our results may be generalizable. Our study was limited in its retrospective nature and its reliance upon medical record and coding data. However, the Regenstrief Institute has a proven track record with this kind of work and the accuracy of the retrieved data has been well documented. ${ }^{15,16}$ Another limitation is that we do not know what information the provider used to aid in the decision to provide the $\mathrm{BMZ}$ injections other than that obtained from the medical record. NICU admission alone does not herald future health, however, it has been demonstrated that infants admitted to the NICU do have increased rates of adverse health outcomes, even when limited to term births. ${ }^{17,18}$ We were unable to determine if any of the women in the ACS group 
had received more than one course of betamethasone. There is also the possibility of these associations being confounded by some unknown variable.

\section{Conclusions}

In conclusion, infants born at term may be at increased risk for NICU admissions and SGA if they were exposed to antenatal corticosteroids during their development. These early neonatal outcomes can be markers for future infant health. As providers have difficulties determining which patients evaluated for threatened preterm birth will actually deliver before term, caution may need to be taken before giving antenatal corticosteroids. 
Table 1: Selected maternal and neonatal characteristics of cohort.

\begin{tabular}{|c|c|c|c|c|}
\hline & $\begin{array}{l}\text { Overall cohort } \\
\qquad(\mathrm{N}=\mathbf{5 3 3 0})\end{array}$ & $\begin{array}{c}\text { BMZ-exposed } \\
(\mathrm{N}=\mathbf{1 4 5 9})\end{array}$ & $\begin{array}{l}\text { BMZ not-exposed } \\
\qquad(\mathrm{N}=\mathbf{3 8 7 1})\end{array}$ & $P$ value \\
\hline \multicolumn{5}{|l|}{ Maternal characteristics } \\
\hline Age (years), mean (SD) & $27.0(5.9)$ & $27.5(6.0)$ & $26.8(5.8)$ & $<.001$ \\
\hline \multicolumn{5}{|l|}{ Ethnicity } \\
\hline Hispanic or Latino & $329(6.2 \%)$ & $90(6.2 \%)$ & $239(6.2 \%)$ & 0.811 \\
\hline Not Hispanic or Latino & $4903(92.0 \%)$ & $1345(92.2 \%)$ & $3558(91.9 \%)$ & \\
\hline Unknown & $98(1.8 \%)$ & $24(1.6 \%)$ & $74(1.9 \%)$ & \\
\hline \multicolumn{5}{|l|}{ Race } \\
\hline Black & $1204(22.6 \%)$ & $292(20.0 \%)$ & $912(23.6 \%)$ & 0.022 \\
\hline White & $3803(71.4 \%)$ & $1077(73.8 \%)$ & $2726(70.4 \%)$ & \\
\hline Other/Unknown & $323(6.1 \%)$ & $90(6.2 \%)$ & $233(6.0 \%)$ & \\
\hline \multicolumn{5}{|l|}{ Insurance } \\
\hline Medicaid/Government & $2941(55.2 \%)$ & $785(53.8 \%)$ & $2156(55.7 \%)$ & 0.125 \\
\hline Private & $2315(43.4 \%)$ & $647(44.3 \%)$ & $1668(43.1 \%)$ & \\
\hline None & $74(1.4 \%)$ & $27(1.9 \%)$ & $47(1.2 \%)$ & \\
\hline Maternal diabetes, yes & $326(6.1 \%)$ & $139(9.5 \%)$ & $187(4.8 \%)$ & $<.001$ \\
\hline Maternal asthma, yes & $144(2.7 \%)$ & $78(5.3 \%)$ & $66(1.7 \%)$ & $<.001$ \\
\hline Maternal hypertensive disorder, yes & $584(11.0 \%)$ & $365(25.0 \%)$ & $219(5.7 \%)$ & $<.001$ \\
\hline EGA when received $\mathrm{BMZ}$ or were & $32.8(4.6)$ & $32.2(3.3)^{\mathrm{a}}$ & $33.0(5.0)$ & \\
\hline \multicolumn{5}{|l|}{ evaluated for threatened preterm labor, } \\
\hline mean $(\mathrm{SD})$ & & & & \\
\hline
\end{tabular}

Neonatal characteristics

EGA at delivery (weeks), mean (SD)

$38.9(1.1)$

$38.4(1.1)$

$39.1(1.1)$

$<.001$ 


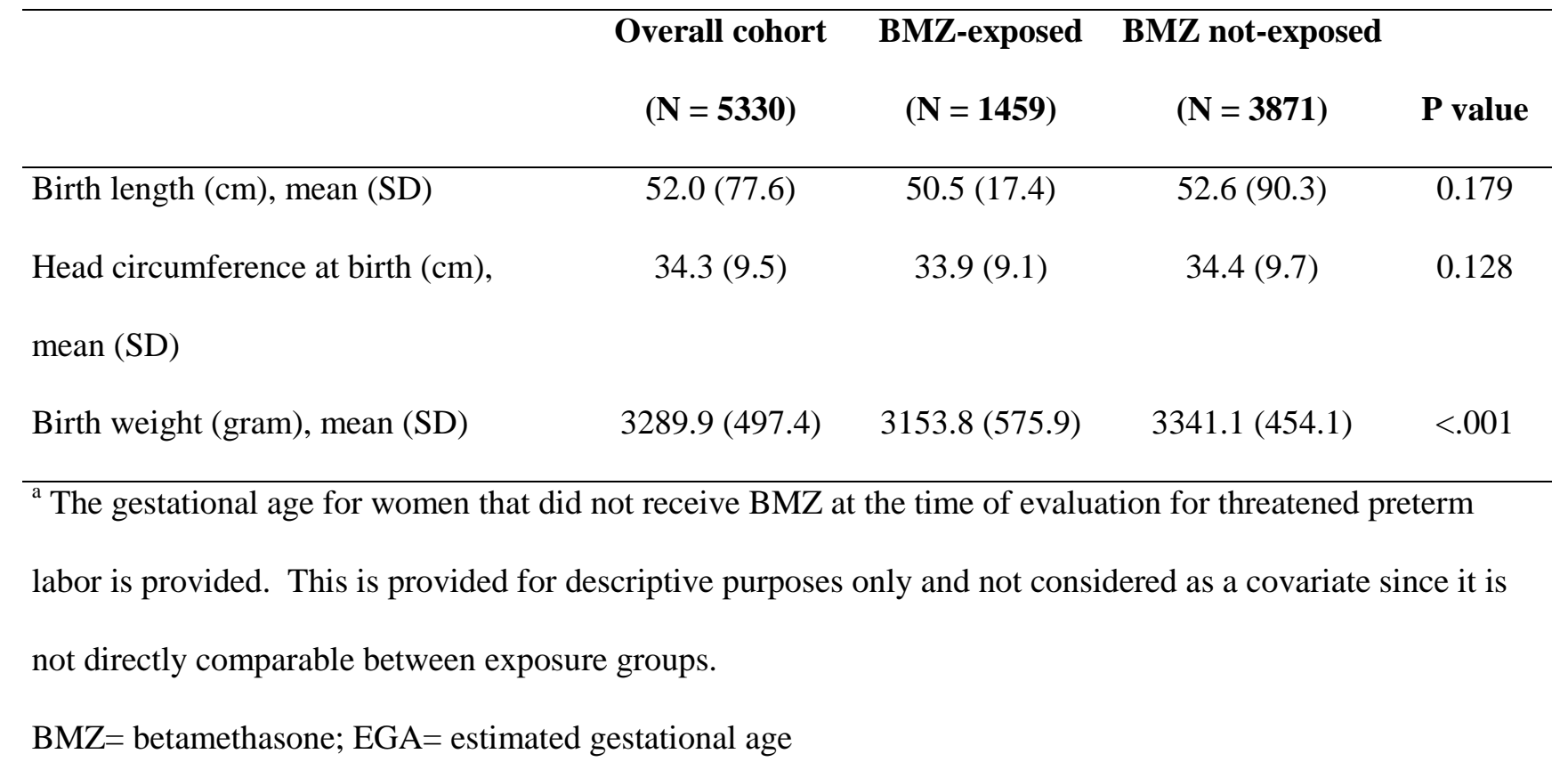


Table 2: Primary and secondary neonatal short-term outcomes

\begin{tabular}{|c|c|c|c|c|c|}
\hline Primary outcomes & $\begin{array}{c}\text { Overall } \\
\text { cohort } \\
(\mathrm{N}=\mathbf{5 3 3 0})\end{array}$ & $\begin{array}{c}\text { BMZ- } \\
\text { exposed } \\
(\mathrm{N}=1459)\end{array}$ & $\begin{array}{l}\text { BMZ not } \\
\text { exposed } \\
(\mathrm{N}=\mathbf{3 8 7 1})\end{array}$ & $\begin{array}{c}\mathbf{P} \\
\text { value }\end{array}$ & \\
\hline Diagnosis of TTN, yes & $215(4.0 \%)$ & $74(5.1 \%)$ & $141(3.6 \%)$ & 0.018 & \\
\hline NICU admission, yes & $426(8.0 \%)$ & $179(12.3 \%)$ & $247(6.4 \%)$ & $<.001$ & \\
\hline Small for gestational age, yes & $\begin{array}{c}\mathrm{N}=4905 \\
699(14.3 \%)\end{array}$ & $\begin{array}{c}\mathrm{N}=1340 \\
263(19.6 \%)\end{array}$ & $\begin{array}{c}\mathrm{N}=3565 \\
436(12.2 \%)\end{array}$ & $<.001$ & \\
\hline Secondary outcomes & & & & $\begin{array}{c}\mathbf{P} \\
\text { value }\end{array}$ & $\begin{array}{c}\mathbf{P} \\
\text { value }^{\mathrm{a}}\end{array}$ \\
\hline Intubation, yes & $42(0.8 \%)$ & $13(0.9 \%)$ & $29(0.7 \%)$ & 0.601 & 0.975 \\
\hline $\begin{array}{l}\text { Hyperbilirubinemia requiring } \\
\text { treatment, yes }\end{array}$ & $806(15.1 \%)$ & $244(16.7 \%)$ & $562(14.5 \%)$ & 0.045 & 0.169 \\
\hline $\begin{array}{l}\text { Treated hypoglycemia newborn, } \\
\text { yes }\end{array}$ & $216(4.1 \%)$ & $96(6.6 \%)$ & $120(3.1 \%)$ & $<.001$ & $<.001$ \\
\hline $\begin{array}{l}\text { Meconium aspiration syndrome, } \\
\text { yes }\end{array}$ & $23(0.4 \%)$ & $5(0.3 \%)$ & $18(0.5 \%)$ & 0.544 & 0.957 \\
\hline
\end{tabular}


Table 3: Logistic regression of primary outcomes, adjusted for covariates.

\begin{tabular}{|c|c|c|c|c|}
\hline & & $\begin{array}{l}\mathrm{N}=5330 \\
(\mathrm{TTN} 4 \%)\end{array}$ & $\begin{array}{c}\mathrm{N}=5311 \\
(\mathrm{NICU} 8 \%)\end{array}$ & $\begin{array}{c}\mathrm{N}=4905 \\
(\mathrm{SGA} 14.3 \%)\end{array}$ \\
\hline \multirow{3}{*}{ Covariate } & \multirow{3}{*}{ Comparison } & Diagnosis of & NICU & \\
\hline & & TTN & admission & SGA \\
\hline & & $\mathrm{aOR}(95 \% \mathrm{CI})$ & aOR $(95 \% \mathrm{CI})$ & $\mathrm{aOR}(95 \% \mathrm{CI})$ \\
\hline \multirow[t]{2}{*}{ BMZ group } & BMZ exposure vs. No & $1.10(0.80$ & $1.49(1.19$, & $1.78(1.48$, \\
\hline & exposure & $1.51)$ & 1.86) & 2.14) \\
\hline \multirow[t]{2}{*}{ Maternal age } & 1-year increase & $0.98(0.96$ & $1.00(0.98$, & $0.97(0.96$ \\
\hline & & $1.01)$ & 1.02) & $0.99)$ \\
\hline \multirow[t]{6}{*}{ Race } & Black vs. White & $1.01(0.72$ & $0.76(0.58$ & $1.80(1.50$ \\
\hline & & 1.42) & $0.99)$ & 2.16) \\
\hline & Other/Unknown vs. White & $1.70(1.04$ & $0.91(0.59$ & 1.69 (1.24, \\
\hline & & 2.77) & $1.40)$ & 2.32) \\
\hline & Black vs. Other/Unknown & $0.59(0.33$ & $0.84(0.52$ & $1.06(0.76$ \\
\hline & & 1.02) & 1.35) & 1.48) \\
\hline \multirow[t]{6}{*}{ Insurance } & Medicaid/Government vs. & $1.30(0.96$ & $1.42(1.14$ & 1.29 (1.07, \\
\hline & Private & $1.76)$ & $1.76)$ & $1.53)$ \\
\hline & None vs. Private & $1.20(0.37$ & $0.20(0.03$ & $1.25(0.64$ \\
\hline & & 3.94) & 1.43) & 2.44) \\
\hline & Medicaid/Government vs. & $1.08(0.33$ & $7.21(0.99$ & $1.03(0.53$ \\
\hline & None & $3.50)$ & $52.46)$ & 1.99) \\
\hline Maternal & Yes vs. No & 1.27 (0.77, & $1.50(1.06$, & $0.92(0.64$ \\
\hline diabetes & & 2.11) & 2.12) & 1.31) \\
\hline Maternal asthma & Yes vs. No & $1.25(0.60$ & $0.91(0.50$ & 1.39 (0.90, \\
\hline
\end{tabular}


Maternal Yes vs. No

hypertensive

disorder

EGA at delivery 1-week increase
$1.10(0.72$

1.68)

$0.74(0.65$

0.84)
$0.92(0.68$,

$0.63(0.57$,

0.70)
$0.86(0.66$

$0.93(0.86$

1.00)

$\mathrm{aOR}=$ adjusted odds ratio; $\mathrm{CI}=$ confidence interval; $\mathrm{BMZ}=$ betamethasone; $\mathrm{EGA}=$ estimated gestational age; $\mathrm{TTN}=$ transient tachypnea of the newborn; $\mathrm{NICU}=$ neonatal intensive care unit; $\mathrm{SGA}=$ small for gestational age. Adjustments made for all other variables in the table. 
Supplemental Table 1: Logistic regression of primary outcomes, adjusted for covariates, not including EGA at delivery

\begin{tabular}{|c|c|c|c|c|}
\hline & & $\begin{array}{l}\mathrm{N}=5330 \\
(\mathrm{TTN} 4 \%)\end{array}$ & $\begin{array}{c}\mathrm{N}=5311 \\
(\mathrm{NICU} 8 \%)\end{array}$ & $\begin{array}{c}\mathrm{N}=4905 \\
(\mathrm{SGA} 14 \%)\end{array}$ \\
\hline \multirow{3}{*}{ Covariate } & \multirow{3}{*}{ Comparison } & Diagnosis of & NICU & \\
\hline & & TTN & admission & SGA \\
\hline & & $\mathrm{aOR}(95 \% \mathrm{CI})$ & $\mathrm{aOR}(95 \% \mathrm{CI})$ & $\mathrm{aOR}(95 \% \mathrm{CI})$ \\
\hline \multirow[t]{2}{*}{ BMZ group } & BMZ exposure vs. No & $1.32(0.97$ & $1.94(1.57$ & $1.87(1.56$ \\
\hline & exposure & 1.79) & $2.40)$ & 2.23) \\
\hline \multirow[t]{2}{*}{ Maternal age } & 1-year increase & $0.99(0.96$ & $1.00(0.99$ & $0.97(0.96$ \\
\hline & & $1.01)$ & $1.02)$ & $0.99)$ \\
\hline \multirow[t]{6}{*}{ Race } & Black vs. White & $1.00(0.72$ & $0.76(0.58$ & $1.80(1.49$ \\
\hline & & $1.41)$ & $0.98)$ & 2.16) \\
\hline & Other/Unknown vs. White & $1.68(1.03$, & $0.90(0.58$ & $1.68(1.23$, \\
\hline & & 2.72) & $1.38)$ & 2.31) \\
\hline & Black vs. Other/Unknown & $0.60(0.35$ & $0.84(0.53$ & $1.07(0.76$ \\
\hline & & $1.03)$ & $1.35)$ & 1.49) \\
\hline \multirow[t]{6}{*}{ Insurance } & Medicaid/Government vs. & $1.32(0.97$ & $1.44(1.15$ & $1.28(1.07$ \\
\hline & Private & 1.79) & 1.79) & $1.54)$ \\
\hline & None vs. Private & $1.15(0.35$ & $0.18(0.03$ & $1.22(0.63$ \\
\hline & & $3.75)$ & 1.34) & 2.39) \\
\hline & Medicaid/Government vs. & $1.15(0.36$ & $7.79(1.08$ & $1.05(0.54$ \\
\hline & None & $3.70)$ & $56.49)$ & 2.03) \\
\hline Maternal & Yes vs. No & $1.44(0.87$ & $1.76(1.25$ & 0.94 (0.66, \\
\hline diabetes & & 2.38) & 2.48) & 1.35) \\
\hline
\end{tabular}


Maternal asthma Yes vs. No

$1.23(0.59$,

2.57)

Yes vs. No

Maternal

hypertensive

disorder

$\mathrm{aOR}=\mathrm{adju}$

gestational age; $\mathrm{TTN}=$ transient tachypnea of the newborn; $\mathrm{NICU}=$ neonatal intensive care unit; $\mathrm{SGA}=$ small for gestational age. Adjustments made for all other variables in the table. 


\section{References:}

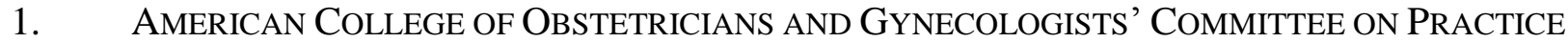
Bulletins- OBSTETRICS. Management of Preterm Labor. Obset Gynecol 2016;4:155-64.

2. Roberts D, DALZIEL, S. Antenatal Corticosteroids for Accelerating Fetal Lung Maturation for Women at Risk of Preterm Birth. Cochrane Database Syst Rev 2006;3.

3. Souza RT GR, LeIte DFB, PASsini R, BAKER P, CECATTI JG. Use of Metabolomics for Predicting Spontaneous Preterm Birth in Asymptomatic Pregnant Women: Protocol for a Systematic Review and Meta-Analysis. BMJ Open 2019;9.

4. Jobe AH GR. Antenatal Corticosteroids: An Assessment of Anticipated Benefits and Potential Risks. Am J Obstet Gynecol 2018;1:62-74.

5. NIXON PA, WASHBURN LK, O'SHEA TM. Antenatal steroid exposure and pulmonary outcomes in adolescents born with very low birth weight. J Perinatol 2013;33:806-10.

6. Bandyopadhyay A, Slaven JE, Evrard C, Tiller C, HaAs DM, TePPer RS. Antenatal corticosteriods decrease forced vital capacity in infants born fullterm. Pediatr Pulmonol 2020.

7. Duryea EL, Hawkins JS, McIntire DD, CASEy BM, LeVeno KJ. A Revised Birth Weight Reference for the United States. Obstetrics \& Gynecology 2014;124:16-22.

8. PEACEMAN AM AW, ThORP JM, Cliver SP, LuKES A, IAMS JD, COULTRIP L, ERIKSEN N, Holbrook RH, Elliott J, Ingardia C, PiEtrantoni M. Fetal fibronectin as a predictor of preterm birth in patients with symptoms: a multicenter trial. Am J Obstet Gynecol 1997;177:13-8.

9. WAPNER RJ, SOROKIN Y, MELE L, et al. Long-term outcomes after repeat doses of antenatal corticosteroids. N Engl J Med 2007;357:1190-8.

10. WAPNER RJ, SOROKIN Y, THOM EA, et al. Single versus weekly courses of antenatal corticosteroids: evaluation of safety and efficacy. Am J Obstet Gynecol 2006;195:633-42.

11. Krispin E, BOROVICH A, HochBERG A, et al. Neonatal outcomes in term pregnancies treated with antenatal corticosteroids for suspected pre-term labor. Archives of Gynecology \& Obstetrics 2019;299:403-09.

12. ShiBATA M, KAJI T, YONETANi N, et al. Effect of prolonged hospitalization on fetal growth in threatened preterm labor. J Med Invest 2019;66:153-56.

13. MANUCK TA. Racial and ethnic differences in preterm birth: A complex, multifactorial problem. Semin Perinatol 2017;41:511-18.

14. Frisbie WP, SONG SE, POWERs DA, StreEt JA. The increasing racial disparity in infant mortality: respiratory distress syndrome and other causes. Demography 2004;41:773-800.

15. Golichowski AM, MCDONALD CJ, TIERNEY WM, et al. Managing perinatal data with the Regenstrief medical record system. J Ambul Care Manage 1992;15:40-53.

16. McDonald CJ, Overhage JM, Tierney WM, et al. The Regenstrief Medical Record System: a quarter century experience. Int J Med Inform 1999;54:225-53.

17. Subedi D, DeBoer MD, Scharf RJ. Developmental trajectories in children with prolonged NICU stays. Arch Dis Child 2017;102:29-34. 
18. Darlow BA, Horwood LJ, Wynn-Williams MB, Mogridge N, Austin NC. Admissions of all gestations to a regional neonatal unit versus controls: 2-year outcome. $\mathrm{J}$ Paediatr Child Health 2009;45:187-93. 